\title{
CALPHAD and the High Entropy Alloy
}

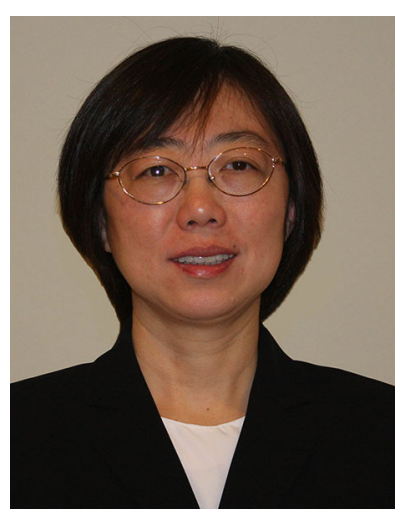

Fan Zhang

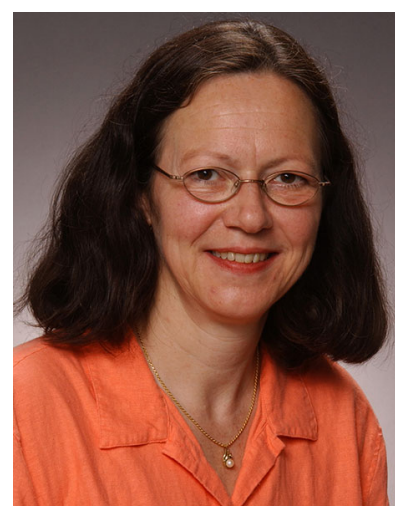

Ursula Kattner

The name of High Entropy Alloy (HEA) first appeared in the paper by Yeh et al. (Advanced Engineering Materials, 6(5) (2004) 299.) published in 2004. The name was given because the authors found that when five or more components with equal/near equal atomic fractions were mixed together, simple solid solution phases instead of multiple intermetallic phases would form. Publications with the key word High Entropy Alloy have increased exponentially since then. Although the design concept based on multiple principal elements indeed opened a wider composition window for the development of new materials with desired properties, experiments have shown that a higher number of components does not necessarily lead to the formation of HEAs. Therefore other factors must be considered when designing such alloys.

Cantor et al. (Materials Science and Engineering A, 375-377 (2004) 213) first investigated an alloy consisting of 20 components in equal atomic proportions (mole fraction of 0.05 each), and then an alloy consisting of 16 elements again in equal atomic proportions (mole fraction of 0.0625 each). Both alloys were found to be multi-phase and brittle, as-cast and after melt spinning. In Otto et al.'s paper (Acta Materialia, 61 (2013), 2628), six alloys were investigated with a base alloy of CoCrFeMnNi. The other five alloys were obtained by replacing one element a time of the base alloy using an element with properties similar to those of the replaced element. The base alloy was found to consist of a single-phase $f c c$, while all the other five alloys consisted of more than one phase. This is the reason why some authors use the term "multi-principal element alloy" instead of HEA for these alloys.

Now the question is how to select the major components to form a HEA? A straight forward way of representing phase stability and phase relationships in an alloy system is with its phase diagram. When a phase diagram is available, one can choose the alloy composition so that a desired microstructure, either a single-phase or a multi-phase mixture, can be developed.

Traditionally, phase diagrams have been determined purely by experimentation which is costly and time consuming when compared with combined experimental and computational methods. While the experimental approach is always necessary and is feasible for the determination of binary and simple ternary phase diagrams, it is less practical for complicated ternaries and quite impractical for higher order systems over a wide range of composition and temperature. Over the past decades the CALPHAD method (Kaufman and Bernstein, Computer Calculation of Phase Diagrams. 1970: New York: Academic Press) has been widely used for the study of phase equilibria of multi-component systems. The essence of this method is to obtain self-consistent thermodynamic descriptions of the lower order binary and ternary systems, in terms of known thermodynamic data measured experimentally and/or calculated theoretically as well as in terms of the measured phase equilibria. The advantage of this method is that the phase diagram and thermodynamic properties are represented by the same "thermodynamic description" (also called "thermodynamic database") of the material system in question. More importantly, on the basis of the known descriptions of the constituent binary and ternary systems, a consistent prediction of higher order systems can be obtained via an extrapolation method.

The CALPHAD method has been successfully applied to the design and development of traditional alloys based on one principal element. Application of this method to the design of alloys with multiple principal elements is more challenging. Traditional alloy design requires a thermodynamic database that covers a limited composition space at the principal-element corner, while design of alloys with multiple principal elements requires a database that is valid in the entire composition range. This database must include full thermodynamic descriptions of all the constituent binaries and ternaries that are consistent with each other. If new phases appear in quaternary systems, then thermodynamic descriptions for the quaternary phases should be developed as well. It requires not only significant efforts on the thermodynamic modeling, but also large amount of experimental data for the constituent binaries and ternaries. This database will certainly need to be validated by experiments and improved when necessary. Development of such a database with 10 or more elements is extremely challenging and time consuming. However, for the purpose of finding a single solid solution phase with high entropy, a two-step approach can be adopted (Calphad, 45 (2014), 1). In the first step, binary phase diagrams, calculated or measured, are used to identify "matching elements" that form a solid solution phase over a wide composition range. In the second step, a thermodynamic database can be developed for a limited number of "matching elements". This database can then be used to identify the composition range where simple single-phase structure may form. With this two-step approach a thermodynamic database with 
limited number of "matching elements" can be developed in a reasonable time frame and this will enable the use of the CALPHAD method as design tool for HEAs or multi-principal element alloys.

\author{
J.-C. Zhao \\ Associate Editor \\ Journal of Phase Equilibria and Diffusion \\ Email: fan.zhang@computherm.com
}

